九州大学学術情報リポジトリ

Kyushu University Institutional Repository

\title{
EM ALGORITHM IN TOMOGRAPHY : A REVIEW AND A BIBLIOGRAPHY
}

Krishnan, T

Indian Statistical Institute

https://doi.org/10.5109/13440

出版情報: Bulletin of informatics and cybernetics. 27 (1), pp.5-22, 1995-03. Research Association of Statistical Sciences

バージョン:

権利関係 : 


\title{
EM ALGORITHM IN TOMOGRAPHY: A REVIEW AND A BIBLIOGRAPHY
}

By

\author{
T. KRISHNaN*
}

\begin{abstract}
In many medical imaging problems such as Positron Emission Tomography (PET), Single Photon Emission Computed Tomography (SPECT) and Transmission Computed Tomography, a lot of projection data for different observation aspects on an object are obtained, allowing reconstruction of the three-dimensional structure of the object from its one and two-dimensional projections. This is basically a problem of analysing incomplete data. Conventional methods such as filtered backprojection (FBP) and Fourier-based methods are deterministic and ignore the statistical elements of the data. Maximum likelihood methods based on suitable statistical models have been found to be satisfactory alternatives to these deterministic methods. The Expectation-Maximisation (EM) algorithm synthesised and presented in a generic form by Dempster, Laird and Rubin in 1977, is an effective iterative method of computing maximum likelihood estimates in a variety of situations, especially in incomplete data problems, where Newton-Raphson types of algorithms are far too cumbersome. In this paper, we review applications of the EM algorithm in tomography and present a bibliography of EM algorithm in tomography.
\end{abstract}

Key Words and Phrases: Positron Emission Tomography (PET), Single Photon Emission Computed Tomography (SPECT), Transmission Computed Tomography, Time-of-Flight PET, X-Ray Computed Tomography, Maximum likelihood estimation, Incomplete data problems, Spatial Poisson process, Gibbs distribution, Smoothed EM, Maximum a posteriori (MAP) estimate

\section{Introduction}

In the last decade or two, there have been exciting developments in computerised tomography towards achieving the goal of identifying abnormal biological activities in connection with given pathologies, directly and as early as possible in the afflicted organisms. Increasing availability of powerful computing facilities and developments in computing techniques such as parallel computing, together with applications of statistical modelling and statistical methods have resulted in availability of imaging techniques fast enough for routine clinical use. A key statistical tool used in these medical image

* Indian Statistical Institute, 203 Barrackpore Trunk Road, Calcutta 700 035, India.

e-mail: krishnan@isical.ernet.in 
reconstruction processes is maximum likelihood estimation or maximum likelihood reconstruction techniques. The EM algorithm which is a generic device to compute maximum likelihood estimates in certain contexts considered to be "incomplete problems" plays a central role in some of these maximum likelihood reconstruction methods. The EM algorithm itself needed several modifications and improvements to be able to be satisfactorily applied to these problems. In this article, we review these applications of the EM algorithm. A recent paper by Kay [29] reviews statistical models used in some medical imaging techniques. We also present a bibliography of EM and related techniques in medical imaging. However, not all items listed in the bibliography have been cited in the text. References for this article which do not involve EM algorithm in medical imaging are presented in a separate list.

\section{Medical Imaging Techniques}

Different imaging techniques such as Transmission Computed Tomography, Emission Computed Tomography (Positron and Single Photon), Nuclear Magnetic Resonance (imaging or in vivo spectroscopy), Digital Subtraction Radiography, Ultrasound and Heavy Ion Radiography are intended to measure different parameters and consequently have different applications. For details, one may see, for instance Brownell, Budinger, Lauterbur and McGeer [85]. It is to be emphasised that it is the parameter measured rather than the image resolution or display quality that is important in a particular disease condition. When it comes to tomographic methods, there are important differences between X-ray transmission tomography and emission tomography, both physical and biological. In transmission tomography, an active experiment is assumed and the object under study is subjected to a predetermined probe radiation. The result of interaction of this probe wave with inhomogeneities of the medium is registered. In emission tomography the object itself is a source of some radiation or some probe radiation sources are introduced into it as in Positron Emission Tomography of the human body. In X-ray transmission tomography, the distribution of tissue densities or attenuation coefficients is sought. In emission tomography, the source and intensity of the gamma radiation emitted by the isotope is sought; effect of attenuation is to be compensated. ET describes regional function and X-ray CT regional structure or anatomy.

Positron Emission Tomography (PET) and Single Photon Emission Computerised Tomography (SPECT) have different underlying physical processes and manners of data collection. In both, a radiopharmaceutical is introduced into the organ. In PET, the resulting radioactive emissions are recorded. In SPECT, the concentration of isotope as a function of the position in patient's body is estimated.

\section{Deterministic and Statistical Methods}

In both transmission (TT) and emission (ET) methods of tomography, a lot of projection data for different observation aspects are obtained, allowing the reconstruction of three-dimensional structure of an object from its one and two-dimensional projections. Special scanning schemes for transmitters and receivers with respect to the object in TT 
and for the object and receivers in ET are used. The geometry of scanning, that is, configuration of the synthesised reception equipment, largely determines quality of image reconstruction, especially in incomplete data situations. The mathematical technique for such a reconstruction is based on inverted-Radon-transform-like methods. A common technique in this context is Filtered Back Projection (FBP), in which the sample projections are first filtered and the image obtained by a discretisation of the continuous FBP method ([87], [88]).

These deterministic methods, although fast and inexpensive as they operate linearly on the data, ignore the stochastic elements of the data and have been found to be not so satisfactory and have well documented deficiencies. They yield fairly accurate reconstructions in situations of high radiation dosage where the photon counts are high and the stochastic nature of the data can be ignored. In scanning protocols where the photon flux is reduced due to either reduced radiation dosage or high level of attenuation, the stochastic nature of the data can no longer be ignored and give rise to severe artifacts in images if conventional methods are used. Thus a statistical approach with ML reconstruction is expected to provide high quality reconstructed images in situations of low photon counts. Chornoboy, Chen, Miller, Miller and Snyder [12] provide examples of EM reconstructed SPECT images which are superior to those produced by deterministic techniques such as Convolution Backprojection (CBP) method.

Attempts to overcome these deficiencies have resulted in more careful statistical modelling of projection data and development of more efficient estimation and reconstruction methods. For PET and SPECT, maximum likelihood (ML) reconstruction was found to provide greater noise suppression, greater edge resolution and less variability in replicates and hence more efficient estimation. They have also resulted in the possibility of using reduced radioactive dosage levels in emission tomography. SPECT systems being less expensive are in routine use more than the more expensive PET scanners.

ML methods based on suitable statistical models have been found to be satisfactory alternatives to these deterministic methods. In medical imaging problems such as Positron Emission Tomography (PET), Single Photon Emission Computed Tomography (SPECT) and Transmission Computed Tomography, maximum likelihood estimates of pixel concentrations or linear attenuation parameters have to be computed. This is difficult because of the large number of parameters involved. Shepp and Vardi [68] used the EM algorithm to solve this maximum likelihood estimation problem.

Since then many variations to the statistical models used and to the EM algorithm for this problem have been developed in order to speed up the computation and to improve the final image obtained.

\section{EM Algorithm}

We now give a quick introduction to the EM algorithm. The EM algorithm (E for Expectation and $M$ for Maximisation), formally stated and studied by Dempster, Laird and Rubin [86], is an elegant and efficient method for maximum likelihood estimation in "incomplete problems". The EM algorithm is effective when for the given problem, 
there is an associated complete problem, for which the maximum likelihood estimation procedure is simpler. Given such a problem, the EM algorithm exploits the simplicity of the complete-problem likelihood to iteratively compute the maximum likelihood estimates for the original incomplete problem. In each iteration of the procedure, there are two steps, viz., the E or the Expectation Step and the M or the Maximisation Step. The Expectation step "completes" the data set using the current parameter values and the observed data; and the Maximisation step computes the maximum likelihood estimates from such completed data and thus produces the parameter estimates for the next cycle. These cycles are repeated until convergence. This method requires less analytical and computational effort than other competing Newton-Raphson-like methods and in many cases easier to implement on a computer than competing methods.

More precisely, let $\phi$ be a vector parameter; let $Y$ be the observed (incomplete) data vector; let $X$ be the complete-data vector and let $Y$ be a function $h(X)$ of $X$. Let $X$ have density $f(x ; \phi)$ and let $Y$ have density $g(y ; \phi) . g$ can be obtained from $f$ by integration. The E-step then consists in finding the conditional expectation $E\left(\ln f(X ; \phi) \mid Y, \phi^{(i)}\right)$, where $\phi^{(i)}$ is the $i^{\text {th }}$ iteration estimate of $\phi$. In the M-step, this conditional expectation is maximised with respect to $\phi$. Under fairly general conditions, this algorithm converges and gives maximum likelihood estimates. The speed of convergence and the simplicity of the computations depend on the complete problem, the relationship between the complete and the incomplete problems and how incomplete the incomplete data set is, relative to the complete data set.

Unlike the scoring method, the EM algorithm however, does not naturally yield the covariance matrix of the estimator. There are methods developed subsequent to Dempster, Laid and Rubin [86] to compute the covariance matrix of the estimators in an integrated fashion from the EM computations.

\section{Statistical Models and Methods in Tomography}

\subsection{Positron Emission Tomography}

The EM algorithm comes in useful in ML estimation of parameters in a computerised image reconstruction process such as Positron Emission Tomography (PET). We give here a very brief and cursory introduction to PET, formulate the statistical model that it gives rise to, and explain how the problem of ML estimation of parameters is solved in the PET model using the EM algorithm.

The main aim of PET is to estimate the spatial distribution of positron emission intensities along a selected plane of a human organ such as the brain. For this, a chemical compound with the desired biological activity is labelled with a radioisotope that decays by emitting a positron. The emitted positron almost immediately combines with an electron and the two are mutually annihilated with emission of two gamma rays. The gamma rays fly off in nearly opposite directions, penetrate the surrounding tissues and are recorded outside the subject by an array of detectors. A mathematical algorithm applied by the computer rapidly reconstructs the spatial distribution of radioactivity for the selected plane on a cathode ray screen. Images are recorded at intervals after 
administration of the chemical compound and can be colour coded to show differences in activity levels from point to point. With suitable interpretations, these images can provide a noninvasive regional assessment of many biochemical processes essential to the organ.

The image forming process has three steps-detection of gamma rays in the positronannihilation process; identification of direction of travel of radiation; and reconstruction of the distribution of radiation into an accurate geometric image.

Since gamma rays fly off in pairs in nearly opposite directions with a random orientation, a large number, say 1000, of pairs of detectors are placed at $180^{\circ}$ around the organ. An emission is reported only if both elements of a pair of detectors sense highenergy protons coincidentally in straight lines. Emissions outside the defined volume are not detected.

In an elegant formulation of the problem, Shepp and Vardi [68], Vardi, Shepp and Kaufman [80] and Lange and Carson [36], model the emissions that occur as a spatial Poisson process in the three-dimensional region concerned with an unknown intensity function. This region is then discretised into pixels and the emission densities $\lambda_{j}$ in the $j=1,2, \ldots, J$ pixels are the parameters. The observations are the counts $n_{i}^{*}, \quad i=1,2, \ldots, I$ in the $I$ detector tubes. Denoting by $a_{i j}$ the conditional probability that emissions from pixel $j$ are detected in tube $i$, we see that $\sum_{i} a_{i j}=1$ and that expected value of $n_{i}^{*}$ is $\sum_{j} a_{i j} \lambda_{j}$. The values of $a_{i j}$ are known from the geometry of the detector tubes.

Many iterative schemes have been suggested for the solution of the likelihood equation given rise to by this model. Of these, the EM algorithm is one of the most appealing ones. We indicate it here. Consider as the complete problem, the problem of ML estimation of $\lambda_{j}$ with the data

$$
n^{* *}=\left\{n_{i j}: i=1,2, \ldots, I ; j=1,2, \ldots, J .\right\},
$$

where $n_{i, j}$ denotes the number of emissions from pixel $j$ recorded in tube $i$. The $\boldsymbol{n}^{* *}$ is now a spatial Poisson point process; the $n_{i j}$ are independent Poisson variables with expectations $n_{j} a_{i j}$. Then it is easy to derive the EM algorithm as the repeated application of

$$
\lambda_{j}^{(t+1)}=\lambda_{j}^{(t)} \sum_{i=1}^{I} \frac{n_{i}^{*} a_{i j}}{\sum_{r=1}^{J} \lambda_{r}^{(t)} a_{i r}} .
$$

Thus the EM algorithm in this case is a repeated application of this M-step in calculating new $\lambda_{j}$ 's from the previous cycle $\lambda_{j}$ 's. There are some difficulties about the convergence of the algorithm and the uniqueness of the MLE for this problem. Indeed, if the number of detector tubes $I$ is less than the number of pixels $J$, the solution will not be unique. Some of these problems are discussed in Green [21] and in Shepp and Vandebei [67].

An algorithm called the Image Space Reconstruction Algorithm (ISRA) is proposed by Daube-Whitherspoon and Muellehner [14] as an alternative to the EM algorithm. 
They point out certain computational advantages of ISRA over the EM algorithm. Titterington [78] shows that this ISRA is a least squares algorithm and that it does not produce estimates of emission densities as good as the EM algorithm in terms of precision. Ollinger [56] proposes a modification of the Convolution Backprojection (CBP) method by incorporating estimation of missing projections. He calls it Iterative ReconstructionReprojection (IRR) method. He shows that this method is an instance of the EM algorithm and further that the standard consistency condition for images needed for the standard deterministic methods can be relaxed to only need the means of the observed data to be consistent and not the data themselves. Thus the algorithm can be used on noisy data such as those encountered in ECT.

\subsection{Single Photon Emission Computed Tomography (SPECT)}

In SPECT, detectors are arranged in a linear array at equally spaced lateral sampling intervals and the detector array can be positioned at any orientation $\theta$ relative to horizontal axis. A rotatable detector $\mathrm{D}$, consisting of collimators collects data at each of $N_{\theta}$ fixed angular positions. Photons that reach the camera surface and are detected generate a measurement point process $N_{m}$ over the product space $D \times \Theta$ where $\Theta=\left\{\theta_{1}, \theta_{2}, \ldots, \theta_{N_{\theta}}\right\}$ is the set of $N_{\theta}$ discrete angular camera positions. The aim of the imaging problem is to estimate the tracer concentration given $N_{m}$ over $D \times \Theta$.

The $N_{m}$ are modelled as independent Poisson variables, with expectation expressed in terms of the attenuated Radon transform averaged over the width of the sampling interval for the detector and its possible orientations, that is, $N_{m i}$ is a Poisson process with intensity

$$
\mu_{m i}=\mu_{m i}(\mu, \theta)=\frac{1}{2 \pi} \int_{\ell_{\theta}^{\perp}(u)} \lambda(x) d x,
$$

where $\ell_{\theta}^{\perp}(u)$ is the line perpendicular to the detector surface that intersects at point $u$, $\lambda(x)$ is the intensity of radioactive decay and is assumed to be proportional to tracer concentration and that single photon emissions are released in an isotropic manner. The constant $\frac{1}{2 \pi}$ shows that only photons directed toward the detector are recorded. EM algorithm for this problem is developed in Miller, Snyder and Miller [53]. Geman and McClure [17] and Green [21] discuss modelling issues, modifications of the EM algorithm and the practicalities involved in SPECT exercises.

\subsection{Time-of-Flight Positron Emission Tomography (TOFPET)}

Time of flight data represent the difference between the times at which two photons created by positron annihilation and propogating in very nearly opposite directions, arrive at opposing detectors. This time difference can be used to estimate the location at which an annihilation occurred along a line connecting the positions of the two detected photons.

This gives rise to a model similar to that of PET. However, the weights $c(x, d)$ are more complicated owing to the need to model attenuation. A modification of the EM algorithm for this problem is carried out in Snyder and Politte [73]. In Chen and 
Metz [9], [10], comparisons of TOFPET algorithms are made and the EM algorithm simplified.

\subsection{X-Ray Computed Tomography}

The primary goal in transmission CT is to reconstruct a two-dimensionale representation of the linear attenuation coefficients $\mu_{i}$ through a cross-section of an object. A set of tubes indexed by $j$ is used whereby $N_{0 j}$ photons are injected as a beam into the tube and $N_{L j}$ photons exit the tube. The remaining photons are absorbed in the attenuation medium of the object, with the $N_{L j}$ photons being recorded by the detectors of the tomographic scanner. We assume that:

1. the $N_{0 j}$ photons constitute a monochromatic X-ray beam;

2. a detector registers a photon only if has left the source in the direction of the detector and has reached the detector without having been absorbed or scattered, so the effects of scatter are ignored;

3. all nonattenuated photons are detected.

Regions are divided into pixels. Pixels are viewed as point samples of a continuum. Each tube is subdivided into $L$ regions of equal length $\Delta$, sufficiently small so that attenuation coefficients can be considered constant. Number of photons that exit region $m$ of tube $j$ is denoted by $N_{m j}$.

The ML problem is that estimating the vector quantity

$$
\mu=\left(\mu_{1}, \mu_{2}, \ldots \ldots\right)^{T}
$$

from projection data

$$
N_{L}=\left(N_{L 1}, N_{L 2}, \ldots, \ldots\right)^{T} \text {. }
$$

We assume that the number of photons injected into each tube is Poisson. The set of injected photons is denoted

$$
N_{0}=\left(N_{01}, N_{02}, \ldots, \ldots\right)^{T}
$$

We assume mean of $N_{0 j}$ to be known. The conditional distribution $N_{L j} \mid N_{o j}$ is modelled as binomial on $N_{o j}$ and

$$
p_{j}=e^{-\Delta \sum_{m=1}^{L} \mu_{m j}^{\prime}},
$$

where $\mu_{m j}^{\prime}$ is the attenuation coefficient of region $m$ of tube $j$.

An application of the steps of the EM algorithm in this case leads in the M-step the following likelihood equations. Here $M_{i j}, N_{i j}$ denote the expected number of photons entering and leaving pixel $j$, respectively for projection $i . J_{k}$ denotes the set of 
projections to which pixel $k$ contributes, $\ell_{i k}$ denotes the length of projection line $i$ that intersects pixel $k$.

$$
\begin{aligned}
0 & =\sum_{i \in J_{k}}-N_{i k} \ell_{i k}+\sum_{i \in J_{k}}\left(M_{i k}-N_{i k}\right) \frac{\ell_{i k} e^{-\ell_{i k} \mu_{k}}}{1-e^{-\ell_{i k} \mu_{k}}} \\
& =\sum_{i \in J_{k}}-N_{i k} \ell_{i k}+\sum_{i \in J_{k}}\left(M_{i k}-N_{i k}\right) \frac{\ell_{i k}}{e^{-\ell_{i k} \mu_{k}}-1}
\end{aligned}
$$

This is a transcendental equation. Lange and Carson [36] discuss a method of solving this equation, by means of simplifying approximations. Browne and Holmes [4] discuss a modification in the EM algorithm by suggesting two complete data sets. We do not go into the details here.

In a different modelling approach to this problem Lei and Sewchand [37], [38] model $Z$ the number of photons counted by the detector (without having been absorbed or scattered) as a Gaussian distribution (as an approximation to the Poisson distribution) truncated to a finite interval $(0, w), w$ depending on the physical settings and conditions of the X-ray production system. They make further assumptions of independence of pixel images if their spatial separation is greater than a certain value and that a group of pixel images to be a spatial stationary random process and ergodic in mean and autocorrelation, if sampled from the same region with the same sampling step. Then they argue that $\operatorname{IMG}(J, K)$ denoting an observed image which has $J$ pixels and $K$ regions are such that IMG in the same $K$ are identical Gaussian and the $K$ image regions compose a Gaussian random field. They develop image reconstruction techniques by EM and CM (Classification-Maximisation) algorithms.

\section{Problems with EM and their solutions}

\begin{tabular}{ll|l}
\hline \multicolumn{2}{c|}{ Problem with EM } & \multicolumn{1}{c}{ Solution } \\
\hline $\begin{array}{l}\text { towards convergence solu- } \\
\text { tion gets noisier and even- } \\
\text { tually gets noisier than data }\end{array}$ & $\begin{array}{l}\text { stop before deterioration or stop after a } \\
\text { certain number of steps; hypothesis testing } \\
\text { approach }\end{array}$ \\
\hline $2 . \quad \begin{array}{l}\text { ill-posed inverse problem; } \\
\text { needs to be regularised }\end{array}$ & $\begin{array}{l}\text { Bayesian techniques; stopping as in (1) } \\
\text { above is an entropic form of regularization }\end{array}$ \\
\hline 3. & $\begin{array}{l}\text { ML reconstruction can suf- } \\
\text { fer from noise and edge } \\
\text { artifacts }\end{array}$ & $\begin{array}{l}\text { method of sieves (a form of regularisation) } \\
\text { for noise; for edge artifacts, use a coarser } \\
\text { resolution in body space obtained by recon- } \\
\text { structing a smoother version of the isotope } \\
\text { concentration function }\end{array}$ \\
\hline $4 . \quad \begin{array}{l}\text { EM slower to converge than } \\
\text { conventional methods }\end{array}$ & $\begin{array}{l}\text { can be implemented to produce restoration } \\
\text { quickly by parallel processors }\end{array}$ \\
\hline $\begin{array}{l}\text { ML has low bias and large } \\
\text { noise }\end{array}$ & $\begin{array}{l}\text { regularisation techniques to stabilise solu- } \\
\text { tion; introduce bias and decrease variation }\end{array}$ \\
\hline
\end{tabular}


In a series of papers [81], [46], [47], [54], the possibility of obtaining visually excellent images by stopping the MLE algorithm using stopping rules based on testing hypotheses is examined.

In some problems, the M-step of the EM algorithm involves solution to transcendental equations. In some problems the EM algorithm is slow to converge, needs large computation time and has non-uniform correction efficiency. Various speeding-up techniques have been proposed. Kaufman [27] suggests a a method which exploits the symmetry of the physical system. Ranganath, Dhawan and Mullani [64] suggest the use of a multigrid system to overcome the speed and non-uniform correction efficiency problems. Tanaka [75] suggests a high frequency enhanced filtered iterative reconstruction method. Lange, Bahn and Little [35] suggest a modified version of the EM algorithm in which each iteration is used to define a direction along which a one-dimensional search for the maximum of the likelihood is made to obtain a new estimate. They call this the EMS algorithm. They also suggest a class of rescaled gradient algorithms. Lewitt and Muehllehner [40] suggest a simpler version of this EMS algorithm, in which the updated image, calculated using the standard EM algorithm, is multiplied in each iteration by an overrelaxation parameter. Rajeevan, Rajagopal and Krishna [63] introduce a class of fast cyclic iterative algorithms suitable for computing MLE of emission densities in ECT. Each iteration in these algorithms involves a few iterations of a standard gradient-based algorithm. The sequence of image estimates thus generated is vector-extrapolated to yield a new image estimate. They present various strategies for vector-extrapolation.

In a Bayesian formulation of the problem Lange, Bahn and Little [35] use a gamma prior pixel by pixel (independently) and estimate parameters by maximum a posteriori probability (MAP), both for transmission and emission tomography. This has the potential for improved images, convergence in fewer iterations, penalisation of the parameters to their lower bounds, converting an underdetermined problem into an overdetermined problem.

In certain problems, correction efficiency of the EM algorithm is nonuniform over the image. Modifications of the EM algorithm such as Smoothed EM, EM-CM, Generalised EM for Bayesian estimation and EM for Maximum a posteriori (MAP) estimation are available.

\section{Bayesian Formulation}

This formulation is due to Besag [2] and Geman and Geman [18]. Let $p(x)$ denote the isotope concentration distribution. We take $p(x)$ to have the form

$$
p(x)=\exp (-U(x, \beta))
$$

the Gibbs distribution. The function $U$ is the energy function associated with the Gibbs distribution.

$$
U(x, \beta)=\sum_{c \in C} V_{c}(x, \beta) .
$$

Here $\beta$ are the parameters of the prior distribution, $C$ denotes a set of cliques and $c$ a particular clique. A most commonly used prior consists of second-order neighbourhoods 
and pairwise interactions between all pairs of neighbouring pixels with an energy function of the form:

$$
U(x, \beta)=\beta \sum_{s \sim r} w_{s r} \phi\left(\left(x_{s}-x_{r}\right) / \delta\right)
$$

where $s \sim r$ means pixels $s$ and $r$ are neighbours, $w_{s r}$ denoting weights reflecting closeness of neighbours ( 1 if horizontal or vertical, $\frac{1}{\sqrt{2}}$ if diagonal, etc., $\delta$ is a scale parameter). Both $\beta, \delta$ are assumed known. Usually the posterior distribution also has a Gibbs distribution with energy function

$$
\ell(x, y)-\beta V(x)
$$

$\ell$ being the loglikelihood and $y$ the observations. This approach can also be considered to be a penalised likelihood approach. Hebert and Leahy [23] develop a GEM algorithm for MAP reconstruction with priors in the form of locally correlated Markov Random Fields using Gibbs functions. This algorithm closely follows the Shepp-Vardi [68] EM algorithm and in fact reduces to it when the prior tends to the uniform distribution. Hebert and Leahy [23] apply their method to single photon emission data. In [19], [20], Gibbs energy functions have been used as prior distributions in SPECT.

Other types of priors with EM approach to Bayesian reconstruction have also been used. Liang and Hart [42] and Hart and Liang [22] used uncorrelated Gaussian and Poisson priors. Liang [41], Liang, Jaszczak and Hart [43] and Liang, Jaszczak and Greer [44] have used uniform and nonuniform probability distributions as priors. Their formulation in some cases leads to explicit EM solutions after some approximations. Levitan and Herman [39] use uncorrelated Gaussian prior and present an explicit algorithm. Lange, Bahn and Little [35] use gamma function priors independent over pixels, as pointed out earlier. Nunez and Llacer [55] use the entropy of the generated image as a prior distribution. They report that their method results in images that do notexhibit deterioration characteristic of MLE reconstructions at a high number of iterations. The EM-based Bayesian reconstruction procedures sometimes perform better than ML EM. Further, in order to develop a closed-formM-step, some modifications are done to the algorithm, which destroys the EM property of the algorithm and may have questionable convergence properties.

\section{Smoothed EM}

The EM solution is generally found to be too rough and noisy. So it is suggested by Vardi, Shepp and Kaufman [80] to use a smoothed version of MLE. For instance, a smoothing matrix may be used as follows:

$$
x_{s}^{\text {new }}=\sum_{r} w_{s r} x_{r}^{\text {old }} \sum_{t} y_{t} \frac{p_{r t}}{\sum_{u} x_{u} p_{u t}} .
$$

Here, $W$ is generally taken to be a row stochastic matrix. This can also be viewed as an approximation to a penalised likelihood approach. Silverman, Jones, Wilson and Nychka [70] suggest a modified EM algorithm by including a smoothing step in each iteration. They relate this method to a penalised likelihood approach. Green [21] suggests in a 
Bayesian approach in which maximum a posteriori probability estimate is proposed, that in the prior, knowledge about the smoothness of the isotope distribution be built in.

There are many other methods suggested in various contexts, many of them being variations and generalisations of the EM algorithm, including some Gibbs sampling methods and other Markov Chain Monte Carlo methods.

\section{Bibliography}

[1] Abdalla, M. and Kay, J.W.: Edge-preserving image restoration. In Stochastic Models, Statistical Methods and Algorithms in Image Analysis, (eds. Barone, P., Frigessi, A. and Piccioni, M.), pages 1-12, New York: Springer-Verlag (1992).

[2] Besag, J.: On the statistical analysis of dirty pictures. Journal of the Royal Statistical Society B, 48 (1986), 259-302.

[3] Bowsher, J.E. and Floyd, C.E., Jr.: Treatment of Compton scattering in maximumlikelihood expectation-maximization reconstructions of Spect images. Journal of Nuclear Medicine, 32 (1991), 1285-1293.

[4] Browne, J.A. and Holmes, T.J. (1992): Developments with maximum likelihood $X$-ray computed tomography. IEEE Transactions on Medical Imaging, 11 (1992), $40-52$.

[5] Byrne, C.L.: Iterative image reconstruction algorithms based on cross-entropy minimization. IEEE Transactions on Image Processing, 2 (1993), 96-103.

[6] Calliol, H., Hillion, A. and Pieczynski, W.: Fuzzy random fields and unsupervised image segmentation. IEEE Transactions on Geoscience and Remote Sensing, 31 (1993), 801-810.

[7] Carson, R.E.: A maximum likelihood method for region of interest evaluation in emission tomography. Journal of Computer Assisted Tomography, 10 (1986), 654663.

[8] Chen, C.T., Johnson, V.E., Wong, W.H., Hu, X.P. and Metz, C.E.: Bayesian image reconstruction in positron emission tomography. IEEE Transactions in Nuclear Science, 37 (1990), 636-641.

[9] Chen, C.-T. and Metz, C.E.: Evaluation and comparison of image reconstruction algorithms for positron emission tomography with time-of-flight information (TOF$P E T)$. Proceedings of the Society of Photo-Optical Instrumentation Engineers, 515 (1984), 388-.

[10] Chen, C.-T. and Metz, C.E.: A simplified EM algorithm for TOFPET. IEEE Transactions in Nuclear Science, 32 (1985), 885-888. 
[11] Chen. L.S.: Solving the attenuation problem in emission computed tomography using homomorphic filters. IEEE Transactions on Acoustics, Speech and Signal Processing, 38 (1990), 714-717.

[12] Chornoboy, E.S., Chen, C.J., Miller, M.I., Miller, T.R. and Snyder, D.L.: An evaluation of maximum likelihood reconstruction for SPECT. IEEE Transactions on Medical Imaging, 9 (1990), 99-110. (Correction: 9, 347-350.)

[13] Chornoboy, E.S., Schramm, L.P. and Karr, A.F.: Maximum likelihood identification of neural point process systems. Biological Cybernetics, 59 (1988), 265-275.

[14] Daube-Witherspoon, M.E. and Muellehner, G.: An iterative image space reconstruction algorithm suitable for volume ECT. IEEE Transactions on Medical Imaging, 5 (1986), 61-66.

[15] Ganti, G. and Mullani, N.: Correction procedures for MGEM algorithm with real data. Journal of Nuclear Medicine, 29 (1988), 774-.

[16] Geman, S.: Statistical methods for tomographic image reconstruction. Bulletin of the International Statistical Institute, LII-4 (1987), 5-21.

[17] Geman, S.: Statistical methods for tomographic image reconstruction. Proceedings of the $46^{\text {th }}$ Session of the International Statistical Institute, Bulletin of the International Statistical Institute, 52 (1987).

[18] Geman, S. and Geman, D.: Stochastic relaxation, Gibbs distribution, and the Bayesian restoration of images. IEEE Transactions on Pattern Analysis and Machine Intelligence, 6 (1984), 721-741.

[19] Geman, S. and McClure, D.E.: Bayesian image analysis: An application to single photon emission tomography. ASA Proceedings of the Statistical Computing Section, (1985), 12-18.

[20] Geman, S. and McClure, D.E.: Statistical methods for tomographic image reconstruction. Bulletin of the International Statistical Institute, LII-4 (1987), 5-21.

[21] Green, P.J.: Bayesian reconstructions from emission tomography data using a modified EM algorithm. IEEE Transactions on Medical Imaging, 9 (1990), 84-93.

[22] Hart, H. and Liang, Z.: Bayesian image processing in two dimensions. IEEE Transactions on Medical Imaging, 6 (1987), 201-208.

[23] Hebert, T. and Leahy, R.: A generalized EM algorithm for 3D Bayesian reconstruction from Poisson data using Gibbs priors. IEEE Transactions on Medical Imaging, 8 (1989), 194-202.

[24] Hebert, T.J. and Gopal, S.S.: The GEM MAP algorithm with 3-D SPECT system response. IEEE Transactions on Medical Imaging, 11 (1992), 81-90. 
[25] Herman, G.T. and Odhner, D.: Performance evaluation of an iterative image reconstruction algorithm for positron emission tomography. IEEE Transactions on Medical Imaging, 10 (1991), 336-345.

[26] Johnson, V, Wong, W.A., Hu, X. and Chen, C.T.: Data augmentation schemes applied to image restoration. Proceedings of the NATO Advanced Study Institute, Povoa, Portugal, September 1988.

[27] Kaufman, L.: Implementing and accelerating the EM algorithm for positron emission tomography. IEEE Transactions on Medical Imaging, 6 (1987), 37-51.

[28] Kaufman, L.: Maximum likelihood, least squares, and penalized least squares for PET. IEEE Transactions on Medical Imaging 12 (1993), 200-214.

[29] Kay, J.: Statistical models for PET and SPECT data. Statistical Methods in Medical Research, 3 (1994), 5-21.

[30] Kay, J.W.: On the convergence of the EMS algorithm. Preprint. 1994.

[31] Kay, J.W.: On combining the ICM and the EM algorithms in image reconstruction. Preprint. 1994.

[32] Kearfott, K.J.: Comment on 'VARdi,Y., L.A.ShepP ANd L.Kaufman's A statistical model for positron emission tomography. Journal of the American Statistical Association, 80, 8-37". Journal of the American Statistical Association, 80 (1985), 26-28.

[33] Kent, J.T. and Wright, C.: Some suggestions for transmission tomography based on the EM algorithm. In Statistical Models, Statistical Methods and Image Analysis, (eds. Barone, P., Frigerri, A. and Piccioni, M.), pages 219-232, Berlin: SpringerVerlag, (1992).

[34] Lange, K.: Convergence of EM image reconstruction algorithms with Gibbs smoothing. IEEE Transactions on Medical Imaging, 9 (1990), 439-446.

[35] Lange, K., Bahn, M. and Little, R.: A theoretical study of some maximum likelihood algorithms for emission and transmission tomography. IEEE Transactions on Medical Imaging, 6 (1987), 106-114.

[36] Lange, K. and Carson, R.: EM reconstruction algorithm for emission and transmission tomography. Journal of Computer Assisted Tomography, 8 (1984), 306-316.

[37] Lei, T. and Sewchand, W.: Statistical approach to X-ray CT imaging and its applications in image analysis-Part I: Statistical analysis of X-ray CT imaging. IEEE Transactions on Medical Imaging, 11 (1992), 53-61.

[38] Lei, T. and Sewchand, W.: Statistical approach to X-ray CT imaging and its applications in image analysis-Part II: A new stochastic model-based image segmentation technique for $X$-ray CT image. IEEE Transactions on Medical Imaging, 11 (1992), $62-69$. 
[39] Levitan, E and Herman, G.T.: A maximum a posteriori probability expectation maximization algorithm for image reconstruction in emission tomography. IEEE Transactions on Medical Imaging, 6 (1987), 185-192.

[40] Lewitt, R.M. and Muehllehner, G.: Accelerated iterative reconstruction for positron emission tomography based on the EM algorithm for maximum likelihood estimation. IEEE Transactions on Medical Imaging, 5 (1986), 16-22.

[41] Liang, Z.: Statistical models of a priori information for image processing. Proceedings of the SPIE Conference on Medical Imaging II. Volume 914 (1988), 677-683.

[42] Liang, Z. and Hart, H.: Bayesian image processing of data from constrained source distributions-non-valued, uncorrelated and correlated constraints. Bulletin of Mathematical Biology, 49 (1987), 51-74.

[43] Liang, Z., Jaszczak, R. and Hart, H.: Study and performance evaluation of statistical methods in image processing. Computers in Biology and Medicine, 18 (1988), 395408.

[44] Liang, Z., Jaszczak, R. and Greer, K.: On Bayesian image reconstruction from projections: Uniform and nonuniform a priori source information. IEEE Transactions on Medical Imaging, 8 (1989), 227-235.

[45] Llacer, J. and Veklerov, E.: The High Sensitivity of the Maximum Likelihood Estimate Method of Tomographic Image Reconstruction. Lawrence Berkeley Laboratory, University of California, Engineering Division Report, LBL 21874 (1987).

[46] Llacer, J. and Veklerov, E.: The use of a stopping rule in iterative image reconstruction. In Proceedings of the Joint Conference of AMA-IMS-SIAM on Spatial Statistical Imaging, Brunswick, ME (1988).

[47] Llacer, J. and Veklerov, E.: Feasible images and practical stopping rules in iterative image reconstruction. IEEE Transactions on Medical Imaging, 8 (1989), 186-193.

[48] Llacer, J., Veklerov, E. and Hoffman, E.J.: On the convergence of the maximum likelihood estimate method of tomographic image reconstruction. Lawrence Berkeley Laboratory, University of California, Engineering Division Report, LBL 21800 (1986).

[49] Llacer, J., Veklerov, E., Coakley, K.J., Hoffman, E.J., and Nunez, J.: Statistical analysis of maximum likelihood estimator images of human brain FDG PET studies. IEEE Transactions on Medical Imaging 12 (1993), 215-231.

[50] McColl, J.H., Holmes, A.P. and Ford, I.: Statistical methods in nueroimaging with particular application in emission tomography. Statistical Methods in Medical Research, 3 (1994), 63-86. 
[51] Miller, M.I. and Butler, C.S.: 3-D maximum a posteriori estimation for single photo emission computed tomography on massively-parallel computers. IEEE Transactions on Medical Imaging 12 (1993), 560-565.

[52] Miller, M., Snyder, D. and Moore, S.: An evaluation of the use of sieves for producing estimates of radioactivity distributions with the EM algorithm for PET. IEEE Transactions in Nuclear Science, 33 (1986), 492-495.

[53] Miller, M., Snyder, D. and Miller, T.: Maximum likelihood reconstruction for single photon emission computed tomography. IEEE Transactions in Nuclear Science, 32 (1985), 769-778.

[54] Nunez, J. and Llacer, J.: Maximum entropy and the concept of feasibility in tomographic image reconstruction. Proceedings of SPIE conference in Medical Imaging, Volume 1090 (1989), 359-372.

[55] Nunez, J. and Llacer, J.: A fast Bayesian reconstruction algorithm for emission tomography with entropy prior converging to feasible images. IEEE Transactions on Medical Imaging, 9 (1990), 159-171.

[56] Ollinger, J.M.: Iterative reconstruction - reprojection and the expectation - maximization algorithm. IEEE Transactions on Medical Imaging, 9 (1990), 94-98.

[57] Phillips, P.R..: Bayesian statistics, factor analysis, and PET images-Part I: Mathematical background. IEEE Transactions on Medical Imaging, 8 (1989), 125-132.

[58] de Pierro, A.R.: On the relation between the ISRA and the EM algorithm for positron emission tomography. IEEE Transactions on Medical Imaging, 12 (1993), 328-333.

[59] Politte, D.G. and Snyder, D.L.: Results of a comparative study of a reconstruction procedure for producing improved estimates of radioactivity distributions in timeof-fight emission tomography. IEEE Transactions in Nuclear Science, 31 (1984), 614-619.

[60] Politte, D.G. and Snyder, D.L.: The use of constraints to eliminate artifacts in maximum likelihood image estimation. IEEE Transactions in Nuclear Science, 35 (1987), 52-56.

[61] Rajeevan, N.: A Stochastic Estimation Approach to Emission Tomography. Ph.D. thesis. Department of Electrical Engineering, Indian Institute of Science, Bangalore, India (1991).

[62] Rajeevan, N.: Vector-extrapolated fast $M L$ algorithms for emission tomography. Proceedings of the $12^{\text {th }}$ Annual International Conference of the IEEE EMBS Society, (Philadelphia) (1990), 370-371. 
[63] Rajeevan, N., Rajgopal, K. and Krishna, G.: Vector-extrapolated fast maximum likelihood estimation for emission tomography. IEEE Transactions on Medical Imaging, 11 (1992), 9-20.

[64] Ranganath, M.V., Dhawan, A.P. and Mullani, N.: A multigrid expectation maximization reconstruction algorithm for positron emission tomography. IEEE Transactions on Medical Imaging, 7 (1988), 273-278.

[65] Rockmore, A.J. and Macovski, A.: A maximum likelihood approach to emission image reconstruction from projections. IEEE Transactions in Nuclear Science, $\mathbf{2 3}$ (1976), 1428-1432.

[66] Rosenqvist, G., Dahlborn, M., Eriksson, L., Bohm, C. and Blomqvist, G.: Quantification of PET data with the EM reconstruction technique. IEEE Transactions in Nuclear Science, 36 (1989), 1113-1116.

[67] Shepp, L.A. and Vandebei, R.J.: New insights into emission tomography via linear programming. NATO Meeting on Formation, Handling and Evaluation of Medical Images. Povoa, Portugal, September 1988.

[68] Shepp, L.A. and Vardi, Y.: Maximum likelihood reconstruction for emission tomography. IEEE Transactions on Medical Imaging, 1 (1982), 113-122.

[69] Shepp, L.A., Vardi, Y., Ra, J.B., Hilal, S.K. and Cho, Z.H.: Maximum likelihood PET with real data. IEEE Transactions in Nuclear Science, 31 (1984), 910-913.

[70] Silverman, B.W., Jones, M.C., Wilson, J.D. \& Nychka, D.W.: A smoothed EM approach to indirect estimation problems, with particular reference to stereology and emission tomography. Journal of the Royal Statistical Society B, 52 (1990), 271-324.

[71] Snyder, D.L.: Utilizing side information in emission tomography. IEEE Transactions in Nuclear Science, 31 (1984), 533-537.

[72] Snyder, D.L. and Miller, M.I.: The use of sieves to stabilise images produced with the EM algorithm for emission tomography. IEEE Transactions in Nuclear Science, 32 (1985), 3864-3872.

[73] Snyder, D.L. and Politte, D.G.: Image reconstruction from list mode data in an emission tomography system having time-of-flight measurements. IEEE Transactions in Nuclear Science, 30 (1983), 1843-1849.

[74] Snyder, D.L., Politte, D.G. and Miller, M.I.: A case study in statistical image processing: Positron Emission Tomography. In Spatial Statistics and Imaging, (ed. Possolo, A.), IMS Lecture Notes-Monograph Series. Hayward, CA: Institute of Mathematical Statistics, (1991). 
[75] Tanaka, E.: A fast reconstruction algorithm for stationary positron emission tomography based on a modified EM algorithm. IEEE Transactions on Medical Imaging, 6 (1987), 98-105.

[76] Tanaka, M. and Katayama, T.: Edge detection and restoration of noisy images by the expectation-maximization algorithm. Signal Processing, 17 (1989), 213-226.

[77] Tanaka, E., Nohara, N., Tomitami, T. and Yamamoto, M.: Utilization of nonnegative constraints in reconstruction of emission tomographs. In Proceedings of the $9^{\text {th }}$ Conference on Information Processing in Medical Imaging, (ed. S.L.Bacharach), Martnus Nijhoff: The Netherlands, (1985).

[78] Titterington, D.M.: On the iterative image space reconstruction algorithm for ECT. IEEE Transactions on Medical Imaging, 6 (1987), 52-56.

[79] Vardi, Y. and Lee, D.: From image deblurring to optimal investments: Maximum likelihood solutions to positive linear inverse problems. Journal of the Royal Statistical Society B, 55 (1993), 569-612.

[80] Vardi, Y., Shepp, L.A. and Kaufman, L.: A statistical model for positron emission tomography (with comments). Journal of the American Statistical Association, 80 (1985), 8-37.

[81] Veklerov, E. and Llacer, J.: Stopping rule for the MLE algorithm based on statistical hypothesis testing. IEEE Transactions on Medical Imaging, 6 (1987), 313-319.

[82] Vermeulen, F.: An improved stochastic reconstruction technique for tomographic imaging. In Proceedings of the $2^{\text {nd }}$ International Symposium on Fundamental Techniques and Progress in Medicine, Liege, Belgium, (1983).

[83] Vishampayan, S., Stamos, J., Mayans, R., Koral, K., Clinthorne, N. \& Rogers, W.L.: Maximum likelihood image reconstruction for SPECT. Journal of Nuclear Medicine, 26 (1985), 20-.

\section{References}

[85] Brownell, G.L., Budinger, T.F., Lauterbur, P.C. and McGeer, P.L.: Positron emission tomography and nuclear magnetic resonance imaging. Science, 215 (1982), 619-626.

[86] Dempster, A.P., Laird, N,M. and Rubin, D.B.: Maximum likelihood from incomplete data via the EM algorithm (with discussion). Journal of the Royal Statistical Society B, 39 (1977), 1-38. 
[87] Gelfand, I.M. and Gindikin, S.G. (Eds.): Mathematical Problems of Tomography. Providence, R.I.: American Mathematical Society, (1990).

[88] Herman, G.T.: Image Reconstruction from Projections. The Fundamentals of Computerized Tomography. New York: Academic Press, (1980).

Received January 2, 1995 